Weekly and daily cycle of alcohol use among the U.S. general population

Wenbin Liang PhD and Tanya Chikritzhs PhD

Wenbin Liang, Statistician

National Drug Research Institute, Curtin University, Perth, Western Australia, Australia

Tanya Chikritzhs, Professor

National Drug Research Institute, Curtin University, Perth, Western Australia, Australia

Correspondence:

Wenbin Liang

National Drug Research Institute, Curtin University,

GPO Box U1987, Perth WA 6845 Australia

Phone: +61 $892661617 \quad$ Fax: +61 892661611

Email: w.liang@curtin.edu.au

Key words: Alcohol, night-time, injury, acute harm 


\title{
Weekly and daily cycle of alcohol use among the U.S. general population
}

\begin{abstract}
Background: Studies such on Alcohol and injuries have defined alcohol-related injury as an injury with a positive self-report of alcohol consumption in the six hours prior to the event. However, there is very limited data on the pattern of alcohol use over time of day and day of week among the general population. The aim of this study is to estimate the rate of alcohol use by time of day, and day of week for the U.S. general adult ( $\geq 18$ years) population.
\end{abstract}

Methods: This study employed the design of a retrospective cohort study using data collected from three waves $(2005-06,2007-08,2009-10)$ of the National Health and Nutrition Examination Survey (NHANES). Incidence rates of overall drinking ( $\geq 10$ grams of alcohol) and incidence rates of heavy drinking ( $\geq 40$ grams of alcohol) were estimated for day of week, and time of day (in hours). Multivariable Poisson regression models were used to investigate the difference between weekend nights and weekday nights.

Results: The incidence rates (95\% confidence interval) of all drinking episodes were 30.5 $(29.2$ - 32.0) per 100 person-days and $24.4(22.8$ - 26.2) per 100 person-days for weekend and the rest of the week, respectively. The incidence rates of heavy drinking episodes were $11.0(10.2-11.9)$ and $7.7(6.8-8.7)$ for weekend and the rest of the week. Multivariable analysis indicated that risks of overall drinking and heavy drinking were significantly higher (18\% and $34 \%$, respectively) during the weekend nights when compared to weekday nights. It was also observed young adults (18-29 years old) were more likely to increase their alcohol use during weekend nights compared to older age groups.

Conclusions: The general US population, especially young adults are exposed to alcohol and its acute effects at a much higher level during the night, and this in-turn increases the risk of alcohol-related injuries during that time. 
Key words: Alcohol, night-time, injury, acute harm 


\section{Introduction}

Alcohol is well established as a major risk factor for injury, and the time interval between exposure to alcohol and injury is important. Experimental studies have demonstrated that increased blood alcohol concentration (BAC) impairs cognitive functioning such as motor control, judgment and alertness in a dose-dependent manner, and because alcohol is metabolized at a constant rate, the risk of injury is likely to be highest during the period before BAC level drops below the low risk level (e.g. legal limit for driving) [1-3]. Many emergency department studies and several meta-analyses have also concluded that exposure to alcohol substantially increases the risk of injury -- particularly injuries occurring within 6 hours of exposure to alcohol [4-7]. Based on agreement between BAC measurement and self-reported alcohol consumption, studies such as the WHO Collaborative Study Group on Alcohol and Injuries have defined alcohol-related injury as an injury with a positive selfreport of alcohol consumption in the six hours prior to the event [4]. In addition, a number of studies have investigated the validity of time of day and day of week as a surrogate measure of alcohol involvement in injuries and road crashes [8-11]. For example in the study by Treno et al, patients admitted to trauma centres during weekend nights were more likely to have a positive BAC result. These observations showed that the risk of injury increases soon after consumption, lasting for a few hours depending on volume of intake, alcohol absorption and metabolism $[12,13]$. Knowing the pattern of alcohol use over time of day and day of week among the general population will aid better understanding of the epidemiology of alcoholrelated injuries and other acute alcohol-caused conditions. This study therefore estimated the rate of alcohol use by time of day, and day of week for the U.S. general population using data collected from three waves $(2005-06,2007-08,2009-10)$ of the National Health and Nutrition Examination Survey (NHANES). 


\section{Method}

This study used data collected from three waves (2005-06, 2007-08, 2009-10) of the National Health and Nutrition Examination Surveys (NHANES). The NHANES surveys employed a multistage sampling design to obtain representative samples of the noninstitutionalized civilian population. The NHANES include interviews collecting information on demographics, dietary and health-related questions. It also includes multiple medical examinations. Details of the NHANES are available on its homepage (http://www.cdc.gov/nchs/nhanes.htm). This study used the alcohol intake data collected from the computer-assisted face-to-face dietary interview component. The face-to-face dietary interview is a 24-hour dietary recall interview conducted in the Mobile Examination Centre (MEC). Information on types and quantity of foods and beverages (including all types of water) consumed during the 24-hour period prior to the interview (midnight to midnight) are coded based on the coding scheme developed by the U.S. Department of Agriculture (USDA).

This study employed the design of a retrospective cohort study. Two levels of alcohol use were defined: 1) 'drinking episode', was defined as having consumed 10 grams or more alcohol on one occasion (i.e. all drinking episodes); and 2) 'heavy drinking episode' was defined as having consumed 40 grams or more alcohol on one occasion. Person-time at risk for a participant was defined as the 24 hours of the dietary recall period of the participant.

Alcoholic beverage consumption records (indicated by "93" in the first two food code digits) were extracted from the dietary datasets. For each record, variables for: the amount of pure alcohol consumed, the starting time (time of day) of consumption, day of the week of consumption and sampling weight were extracted. Demographic information including age, gender, race, US citizenship status, education level and marital status were extracted from the 
demographic datasets and were matched with the alcoholic beverage consumption records based on unique participant identification (ID) numbers. Based on the dietary dataset, a baseline dataset was prepared to account for the amount of person-time at risk. Alcohol consumption records together with demographic information were then matched with the baseline dataset using participant ID and starting time of drinking episode.

\section{Data analysis}

In descriptive analysis, incidence rates of overall drinking ( $\geq 10$ grams of alcohol) and incidence rates of heavy drinking ( $\geq 40$ grams of alcohol) were estimated for day of week, and time of day (in hours). In the multivariable analysis, Poisson regression models were used to investigate the difference between weekend nights and weekday nights. Weekend nights were defined as 17:00 to 0:59 of the next day for Friday, Saturday and Sunday, and weekday nights were defined as 17:00 to 0:59 of the next day for the remaining days of the week. Analysis was further performed for weekday nights and weekend nights separately to compare the difference in rate of heavy drinking between age groups. Demographic characteristics described above were controlled for in the multivariable analysis while sampling weights provided with the dataset were used in all analyses. This study included adults aged 18 years or older at the time of interview. Statistical analysis software package STATA version 12 was used to perform all data analysis.

\section{Results}

The numbers of participants included in this study were 5055, 5682 and 6032 from the 200506, 2007-08 and 2009-10 NHANES, respectively. This totals to 16,769 person-days at risk with 4032 drinking episodes ( $\geq 10$ grams of alcohol) and 1428 heavy drinking episodes $(\geq 40$ 
grams of alcohol) observed. The overall incidence rates (95\% confidence interval) of any drinking episode and heavy drinking episode were 27.0 (25.9-28.2) per 100 person-days and $9.1(8.5-9.8)$ per 100 person-days, respectively. The rates for all drinking episodes and heavy drinking episodes by day of week as well as time of the day are shown in Figures (1-4). As shown in Figure 1, the risk of any drinking episode was higher on the weekend (Friday, Saturday and Sunday) compared to the other days of the week. The incidence rates $(95 \%$ confidence interval) of all drinking episodes were 30.5 (29.2 - 32.0) per 100 person-days and $24.4(22.8$ - 26.2) per 100 person-days for weekend and the rest of the week, respectively. Similar but more notable differences between weekends and the rest of the week were observed for heavy drinking episodes (Figure 2). The incidence rates of heavy drinking episodes were $11.0(10.2-11.9)$ and $7.7(6.8-8.7)$ for weekend and the rest of the week. The pattern of risk by time of day indicated that the majority of drinking episodes started between 12.00 noon and midnight; increasing from 12.00 noon to 19:00, peaking between 18:00 and 20:00 and then decreasing from 21:00 to midnight (Figures 3 and 4).

Multivariable analysis indicated that risk of overall drinking ( $\geq 10$ grams of alcohol) was slightly but significantly higher $(18 \%)$ during the weekend nights when compared to weekday nights. There was a significant $34 \%$ increase in the risk of heavy drinking ( $\geq 40$ grams of alcohol) during weekend nights. As expected, males were more likely to have consumed any alcohol compared to females, while no significant differences between age groups was found except for those aged 70 years and older who had a significantly lower risk of drinking (Table 1). When weekday nights and weekends were analysed separately for heavy drinking, compared to the youngest adults (19-29 years), participants who were (30-49 years) were more likely to drink heavily during weekday nights but not weekend nights, and participants 50-69 years were less likely to drink heavily during weekend nights compared to the youngest age group (Table 2). 


\section{Discussion}

This study investigated the pattern of overall drinking and heavy drinking by day of week and time of day among the U.S. population. To the best of our knowledge, this is the first study that illustrates how alcohol use changes throughout the seven-day week cycle and the 24 hour daily cycle at a population level. Most drinking occasions started at night, indicating that population exposure to alcohol and its acute effects are likely to be much higher during the night compared to daytime hours. This observation concurs with findings from previous studies which suggest that alcohol-related injuries and other alcohol-related harms more commonly occur at night in the United States $[8-10,14,15]$ and in other countries [16-20]. It should be noted that the information on the time of cessation of a drinking episode or the exact time when the last drink was consumed was not available for this study. However, based only on the starting time for heavy drinking episodes (Figure 4), and the six-hour rule used to defined alcohol-related injury in ED studies [4], most of the acute effects of alcohol exposure would tend to occur within the period from about $7 \mathrm{pm}$ until 1:00am. It was also observed that heavy drinking was more likely to occur during weekend nights, and young adults (18-29 years old) were even more likely to increase their alcohol use during weekend nights compared to older age groups. These observations are consistent with the findings from previous studies which have shown alcohol-related injuries to be more common during weekend nights and more likely to affect younger people [8-10, 21, 22].

There are a number of strengths to this study including that: it was based on data collected from a large representative general population sample; information on alcohol use was collected via a validated 24 hour dietary recall interview (which aimed to reduce recall bias); and, demographic characteristics were controlled for in analysis. However, as indicated above, 
it was a limitation that there was no information on the time when a drinking episode ended and therefore we were unable to determine total length of drinking episodes. It is also possible that alcohol use per episode may be under reported. Although potential for recall bias was minimised by asking about use in the past 24 hours, there was no blood testing to confirm accuracy and therefore some degree of recall bias is likely. This study is based on a sample of the general U.S. population, and the weekly cycle and daily cycle of alcohol use may vary in other countries.

\section{Conclusion}

Among the general US population alcohol consumption largely occurs during night-time hours, especially on weekends. Young adults were more likely to increase their drinking frequency during weekend nights. This suggests that the population at large is exposed to alcohol and its acute effects at a much higher level during the night, and this in-turn increases the risk of alcohol-related injuries during that time. 


\section{Acknowledgement}

The National Drug Research Institute at Curtin University is supported by funding from the Australian Government under the Substance Misuse Prevention and Service Improvement

Grants Fund. The funder has no involvement in the study design; collection, analysis and interpretation of data; the writing of the manuscript; the decision to submit the manuscript for publication. 


\section{Reference}

1. Peterson, J.B., Rothfleisch, J., Zelazo, P.D., and Pihl, R. (1990) Acute alcohol intoxication and cognitive functioning. Journal of Studies on Alcohol and Drugs, 51(2), 114.

2. Calhoun, V.D. and Pearlson, G.D. (2012) A selective review of simulated driving studies: Combining naturalistic and hybrid paradigms, analysis approaches, and future directions. NeuroImage, 59(1), 25-35.

3. Meda, S.A., Calhoun, V.D., Astur, R.S., Turner, B.M., Ruopp, K., and Pearlson, G.D. (2009) Alcohol dose effects on brain circuits during simulated driving: An fMRI study. Human Brain Mapping, 30(4), 1257-1270.

4. WHO Collaborative Study Group on Alcohol and Injuries, WHO collaborative study on alcohol and injuries: final report. 2007, Geneva: Department of Mental Health and Substance Abuse, Department of Injuries and Violence Prevention, World Health Organization.

5. Borges, G., Cherpitel, C., Medina-Mora, M.E., Mondragón, L., and Casanova, L. (1998) Alcohol consumption in emergency room patients and the general population: a population-based study. Alcoholism: Clinical and Experimental Research, 22(9), 1986-1991.

6. Cherpitel, C.J., Ye, Y., Bond, J., and Borges, G. (2003) The causal attribution of injury to alcohol consumption: a cross-national meta-analysis from the Emergency Room Collaborative Alcohol Analysis Project. Alcoholism: Clinical And Experimental Research, 27(11), 1805-1812.

7. Zeisser, C., Stockwell, T.R., Chikritzhs, T., Cherpitel, C., Ye, Y., and Gardner, C. (2013) A Systematic Review and Meta-Analysis of Alcohol Consumption and Injury 
Risk as a Function of Study Design and Recall Period. Alcoholism: Clinical and Experimental Research, 37, E1-E8.

8. Treno, A.J. and Holder, H.D. (1997) Measurement of Alcohol-Involved Injury in Community Prevention: The Search for a Surrogate III. Alcoholism: Clinical and Experimental Research, 21(9), 1695-1703.

9. Heeren, T., Smith, R.A., Morelock, S., and Hingson, R.W. (1985) Surrogate measures of alcohol involvement in fatal crashes: Are conventional indicators adequate? Journal of Safety Research, 16(3), 127-134.

10. Voas, R.B., Romano, E., and Peck, R. (2009) Validity of surrogate measures of alcohol involvement when applied to nonfatal crashes. Accident Analysis \& Prevention, 41(3), 522-530.

11. Young, D.J., Stockwell, T., Cherpitel, C.J., Ye, Y., Macdonald, S., Borges, G., et al. (2004) Emergency room injury presentations as an indicator of alcohol-related problems in the community: a multilevel analysis of an international study. Journal of Studies on Alcohol and Drugs, 65(5), 605.

12. Martin, E., Moll, W., Schmid, P., and Dettli, L. (1984) The pharmacokinetics of alcohol in human breath, venous and arterial blood after oral ingestion. European Journal of Clinical Pharmacology, 26(5), 619-626.

13. Jones, A.W. and Andersson, L. (2003) Comparison of ethanol concentrations in venous blood and end-expired breath during a controlled drinking study. Forensic Science International, 132(1), 18-25.

14. Levy, D.T., Mallonee, S., Miller, T.R., Smith, G.S., Spicer, R.S., Romano, E.O., et al. (2004) Alcohol involvement in burn, submersion, spinal cord, and brain injuries. Medical Science Monitor, 10(1), CR17-CR24. 
15. Zador, P.L., Krawchuk, S.A., and Voas, R.B. (2000) Alcohol-related relative risk of driver fatalities and driver involvement in fatal crashes in relation to driver age and gender: an update using 1996 data. Journal of Studies on Alcohol, 61(3), 387-395.

16. Liang, W. and Chikritzhs, T. (2011) Revealing the link between licensed outlets and violence: counting venues versus measuring alcohol availability. Drug and Alcohol Review, 30(5), 524-535.

17. Wells, S. and Graham, K. (2003) Aggression involving alcohol: relationship to drinking patterns and social context. Addiction, 98(1), 33-42.

18. Chikritzhs, T. and Stockwell, T. (2007) The impact of later trading hours for hotels (public houses) on breath alcohol levels of apprehended impaired drivers. Addiction, 102(10), 1609-1617.

19. Keall, M.D., Frith, W.J., and Patterson, T.L. (2004) The influence of alcohol, age and number of passengers on the night-time risk of driver fatal injury in New Zealand. Accident Analysis \& Prevention, 36(1), 49-61.

20. Hughes, K., Anderson, Z., Morleo, M., and Bellis, M.A. (2008) Alcohol, nightlife and violence: the relative contributions of drinking before and during nights out to negative health and criminal justice outcomes. Addiction, 103(1), 60-65.

21. Cherpitel, C.J., Parés, A., and Rodés, J. (1993) Prediction of alcohol-related casualties in the emergency room: a U.S.-Spain comparison. Journal of Studies on Alcohol, $54(3), 308-314$

22. Mäkelä, P., Martikainen, P., and Nihtilä, E. (2005) Temporal variation in deaths related to alcohol intoxication and drinking. International Journal of Epidemiology, 34(4), 765-771. 
Table 1 Risk difference of all drinking episodes ( $\geq 10$ grams of alcohol per occasion) and heavy drinking episodes ( $\geq 40$ grams of alcohol per occasion) between weekday nights and weekend nights*

\begin{tabular}{|c|c|c|c|c|c|c|}
\hline \multirow{2}{*}{ Weekday nights } & \multicolumn{3}{|c|}{ All drinking episodes } & \multicolumn{3}{|c|}{ Heavy drinking episodes } \\
\hline & $\begin{array}{l}\mathrm{IRR}^{\wedge} \\
1.00\end{array}$ & \multicolumn{2}{|c|}{$\begin{array}{l}95 \% \text { Confidence } \\
\text { Interval }\end{array}$} & $\begin{array}{l}\mathrm{IRR}^{\wedge} \\
1.00\end{array}$ & \multicolumn{2}{|c|}{$\begin{array}{l}\text { 95\% Confidence } \\
\text { Interval }\end{array}$} \\
\hline Weekend nights & 1.18 & 1.08 & 1.29 & 1.34 & 1.13 & 1.57 \\
\hline \multicolumn{7}{|l|}{ Gender } \\
\hline Male & 1.00 & & & 1.00 & & \\
\hline Female & 0.53 & 0.48 & 0.58 & 0.31 & 0.25 & 0.37 \\
\hline \multicolumn{7}{|l|}{ Age group } \\
\hline $18-29$ & 1.00 & & & 1.00 & & \\
\hline $30-39$ & 1.09 & 0.93 & 1.29 & 1.20 & 0.93 & 1.55 \\
\hline $40-49$ & 1.13 & 0.95 & 1.34 & 1.23 & 0.93 & 1.62 \\
\hline $50-59$ & 1.02 & 0.85 & 1.21 & 0.88 & 0.65 & 1.18 \\
\hline $60-69$ & 1.00 & 0.83 & 1.21 & 0.82 & 0.59 & 1.15 \\
\hline $70+$ & 0.69 & 0.57 & 0.84 & 0.30 & 0.20 & 0.44 \\
\hline \multicolumn{7}{|l|}{ Race } \\
\hline Mexican American & 1.00 & & & 1.00 & & \\
\hline Other Hispanic & 1.18 & 0.95 & 1.45 & 0.86 & 0.61 & 1.21 \\
\hline Non-Hispanic White & 1.55 & 1.32 & 1.82 & 1.43 & 1.12 & 1.82 \\
\hline Non-Hispanic Black & 1.03 & 0.86 & 1.23 & 0.95 & 0.73 & 1.25 \\
\hline Other Race & 0.80 & 0.61 & 1.06 & 0.75 & 0.46 & 1.22 \\
\hline \multicolumn{7}{|l|}{ Citizenship Status } \\
\hline Citizen & 1.00 & & & 1.00 & & \\
\hline Not a citizen of the US & 0.93 & 0.76 & 1.13 & 0.72 & 0.52 & 1.01 \\
\hline \multicolumn{7}{|l|}{ Education Level } \\
\hline Less Than 9th Grade & 1.00 & & & 1.00 & & \\
\hline 9-11th Grade & 1.30 & 1.02 & 1.66 & 1.53 & 1.05 & 2.23 \\
\hline High School Grad/GED or Equivalent & 1.35 & 1.07 & 1.70 & 1.32 & 0.91 & 1.89 \\
\hline Some College or AA degree & 1.68 & 1.34 & 2.11 & 1.29 & 0.90 & 1.85 \\
\hline College Graduate or above & 2.07 & 1.65 & 2.60 & 1.64 & 1.14 & 2.36 \\
\hline \multicolumn{7}{|l|}{ Marital Status } \\
\hline Married & 1.00 & & & 1.00 & & \\
\hline Widowed & 0.85 & 0.67 & 1.08 & 1.10 & 0.65 & 1.85 \\
\hline Divorced & 1.22 & 1.04 & 1.43 & 1.53 & 1.16 & 2.01 \\
\hline Separated & 1.43 & 1.06 & 1.94 & 3.13 & 2.04 & 4.78 \\
\hline Never married & 1.13 & 0.98 & 1.31 & 1.62 & 1.27 & 2.06 \\
\hline Living with partner & 1.15 & 0.96 & 1.38 & 1.44 & 1.09 & 1.90 \\
\hline
\end{tabular}


$<20$ years old (Marital status not asked)

0.52

0.38

0.71

0.74

0.47

1.16

Year of survey

2005-06

$1.00 \quad 1.00$

2007-08

0.87

0.77

0.99

0.89

2009-10

1.06

0.94

1.19

1.07

0.72

1.10

*Models controlled for all factors listed in the table

0.87

1.31

^Incidence rate ratio 
Table 2 Risk difference of heavy drinking episodes ( $\geq 40$ grams of alcohol per occasion) between age groups stratified for weekday/weekend nights*

\begin{tabular}{|c|c|c|c|c|c|c|}
\hline \multirow{3}{*}{ Age grou } & \multicolumn{3}{|c|}{ Weekday nights } & \multicolumn{3}{|c|}{ Weekend nights } \\
\hline & $\mathrm{IRR}^{\wedge}$ & \multicolumn{2}{|c|}{$\begin{array}{l}\text { 95\% Confidence } \\
\text { Interval }\end{array}$} & $\mathrm{IRR}^{\wedge}$ & \multicolumn{2}{|c|}{$\begin{array}{l}95 \% \text { Confidence } \\
\text { Interval }\end{array}$} \\
\hline & & & & & & \\
\hline $18-29$ & 1.00 & & & 1.00 & & \\
\hline $30-39$ & 1.53 & 0.97 & 2.42 & 0.93 & 0.71 & 1.20 \\
\hline $40-49$ & 1.55 & 0.93 & 2.59 & 1.00 & 0.76 & 1.31 \\
\hline $50-59$ & 1.09 & 0.66 & 1.81 & 0.70 & 0.50 & 0.98 \\
\hline $60-69$ & 1.17 & 0.69 & 1.99 & 0.53 & 0.34 & 0.81 \\
\hline $70+$ & 0.38 & 0.21 & 0.69 & 0.22 & 0.12 & 0.40 \\
\hline
\end{tabular}

*Models controlled for all potential confounding factors listed in Table 1

$\wedge$ Incidence rate ratio 
Figure 1: Incidence rate of drinking episodes ( $\geqslant 10$ grams of alcohol per occasion) by day of week

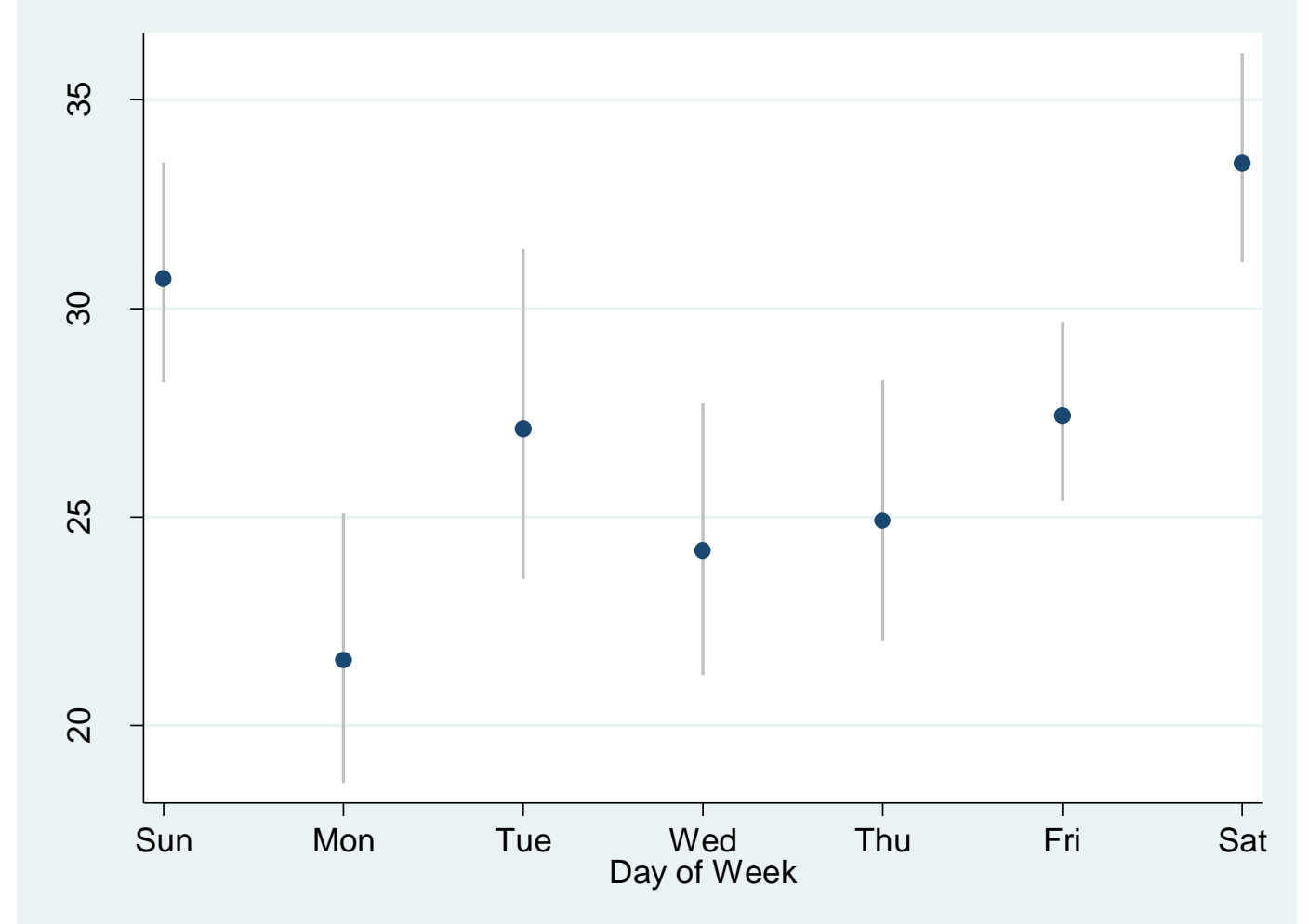

* per 100 person-days, Range indicates $95 \%$ confidence interval 
Figure 2: Incidence rate of heavy drinking episodes ( $\geqslant 40$ grams of alcohol per occasion) by day of week

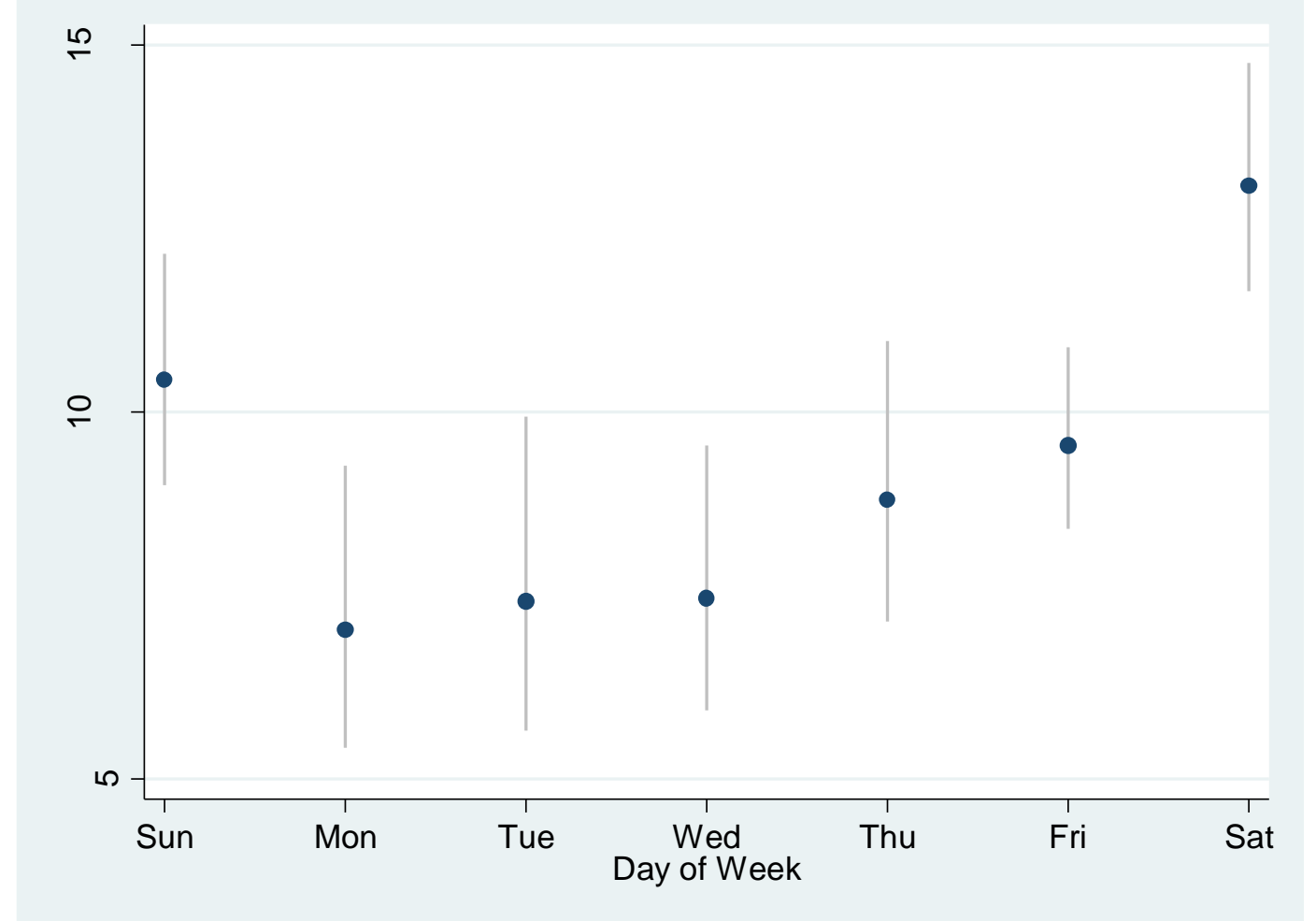

*per 100 person-days, Range indicates $95 \%$ confidence interval 
Figure 3 Incidence rates of drinking episodes ( $\geqslant 10$ grams of alcohol per occasion) by time of day

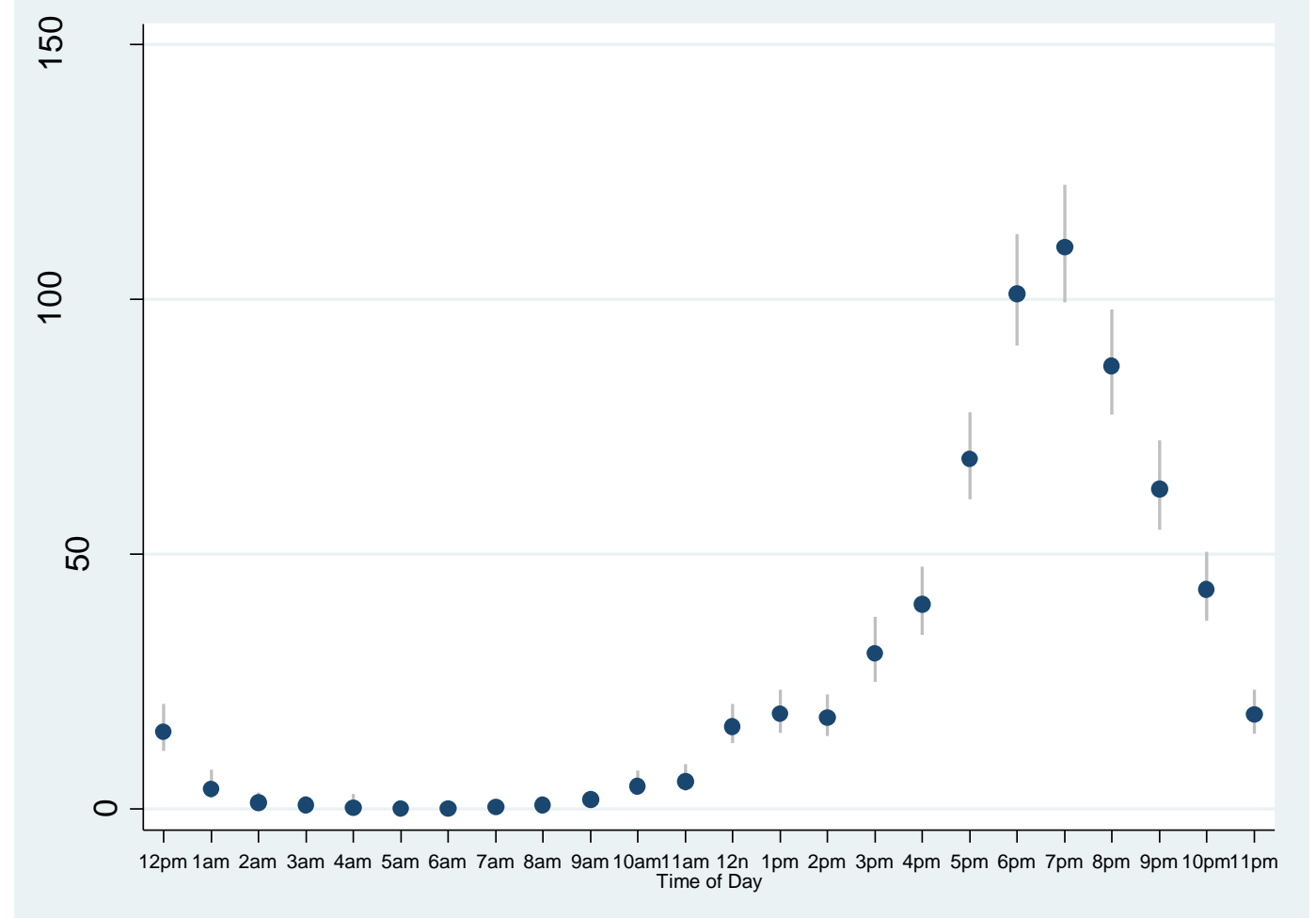

*per 100 person-days, Range indicates $95 \%$ confidence interval 
Figure 4 Incidence rates of heavy drinking episodes ( $\geqslant 40$ grams of alcohol per occasion) by time of day

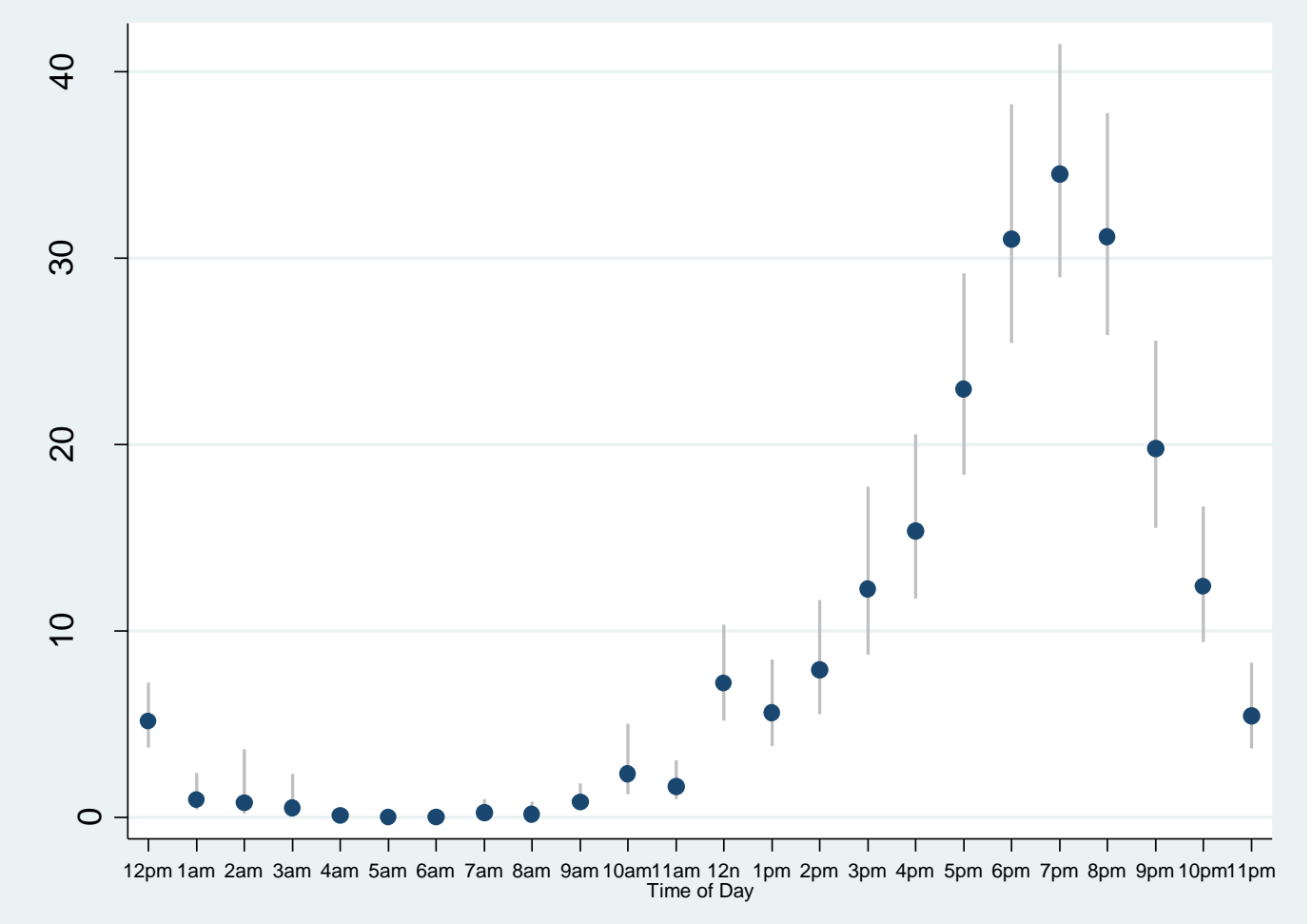

*per 100 person-days, Range indicates $95 \%$ confidence interval 